\title{
TEMPERATURE-FREQUENCY CONTROLLING THE CHARACTERISTICS OF A PULSATING BROWNIAN RATCHET WITH SLIGHTLY FLUCTUATING POTENTIAL ENERGY
}

\author{
I. V. SHAPOCHKINA ${ }^{A}$, T. YE. KOROCHKOVA ${ }^{B}$, V. M. ROZENBAUM ${ }^{B}$, A. S. BUGAEV ${ }^{C}$, L. I. \\ TRAKHTENBERG ${ }^{C, D}$
}

\begin{abstract}
${ }^{a}$ Department of Physics, Belarusian State University, Prospekt Nezavisimosti 4, Minsk 220050, Belarus

${ }^{b}$ Chuiko Institute of Surface Chemistry, National Academy of Sciences of Ukraine, Generala Naumova str. 17, Kiev 03164, Ukraine

${ }^{c}$ N.N. Semenov Federal Research Center for Chemical Physics RAS, 119991 Moscow, Russian Federation

${ }^{d}$ Lomonosov Moscow State University, Vorobyovy Gory, Moscow, 119991, Russian Federation
\end{abstract}

Within the approximation of slight fluctuations of the nanoparticle potential energy, we developed a method for calculating the characteristics of a Brownian ratchet (a complex nonlinear system capable of extracting useful work from unbiased nonequilibrium fluctuations). The method is suitable for studying the mechanisms and modes of functioning of artificial nanomotors. Unlike the effort-consuming obtaining and applying for this studying the Green's functions of the coordinate representation which describe diffusion in the stationary component of the potential, the proposed method operates with the Fourier representation of both the control and desired functions. That allows calculating the Green's functions as inverse matrices in the space of Fourier harmonics and finding the average velocity of a Brownian ratchet with an arbitrary spatial and temporal dependence of the potential energy. To illustrate the method, an analysis has been performed of the functioning of a ratchet in which the directional motion of nanoparticles arises due to small stochastic fluctuations of an asymmetric sawtooth potential profile with an arbitrary barrier-height-to-thermal-energy ratio. It is shown that, with a harmonic coordinate dependence of these fluctuations, a change in the direction and intensity of the ratchet effect is controlled not only by tuning the magnitude of their phase shift relative to the sawtooth potential (the fact revealed before in the high-temperature approximation), but also by changing the temperature and the frequency of fluctuations. The nontrivial dependences of the ratchet velocity on the geometric, frequency, and energy parameters of the system are obtained by numerical implementing the proposed calculation method.

\section{INTRODUCTION}

Controlling of nanoscaled processes is possible through turning various parameters, among which temperature plays a special role. The currently well-known nonlinear systems, called Brownian motors or ratchets [1-6] are not an exception here: temperature changes can lead to changes in the hierarchies of both characteristic times and energies of ratchet systems and thus transfer them to a fundamentally different operating mode up to the elimination of the effect [7-10]. The same result can arise from changes in the frequency of the potential energy of a Brownian motor [1-3,9-11]. Thought the term "Brownian motor" (or "Brownian ratchet") was first invented to illustrate the impossibility of spontaneous emergence of directed motion from equilibrium fluctuations, later it has already been adapted or even reinvented to predict the possibility and explain the mechanism of the appearance of directed motion as a result of rectification of unbiased nonequilibrium fluctuations in nanoscaled nonlinear systems, natural 
or artificial [1,4], under the broken spatial and/or temporal symmetry [12-16]. At present, the controlled ratchet effect widens the set of new methods for controlling the nanoparticle transport which are both already successfully competing [17-20] and will compete or be combined with traditional methods [6]. For that reason, the following problems seem to be actual not only in theoretical, but firstly in an applied context: (i) finding the complete set of control parameters of ratchet systems, (ii) structuring the ranges of their values, operation modes, and responses of ratchets to changes in these values $[1,3,5,6,21]$, (iii) methods for obtaining analytical results and recommendations for using them. As one of the applications of the ratchet theory, we can mention biological protein motors [22]. Another one is molecular machines created either by means of protein engineering [23] to imitate natural objects (e.g., artificial protein motors [24,25], synthetic molecular rotational and translation motors, [26-28]) or on the basis of other ideas, not inspired by protein objects, as, for example, solid-state ratchets [29-34], ratchets on colloidal particles, etc. [35-37] (see also the review of experimental works in [3]).

The source of nonequilibrium fluctuations creating a ratchet potential can be electric and magnetic fields, optical tweezers, channels of a special shape, etc. $[4,17,18,38-40]$. In most ratchet systems, regardless of the source of nonequilibrium fluctuations of characteristics of a system itself or external fields supplying energy to the system, thermal noise plays a fundamental or essential, for functioning of such nanomachines, role $[7,17,18,41,42]$. Chaotic motion caused by thermal noise allows a nanosystem to overcome potential barriers or determines the purely diffusion stage of motion in a cyclic process of changing the state of the system. In protein motors, the diffusion stage ("turning off the potential" that is switching it to the "off" state) means the detaching the head of a motor protein from a substrate (track) [22, 43, 44]. Due to the latter, it is the stochastic (thermal) effect of the environment that makes the motor head diffusely move in space, so that its displacement relative to the middle of the period of an asymmetric potential ("switched on" with a subsequent change in the protein configuration followed by the increased coupling of the head with the track) leads to a higher probability to diffuse to the nearest neighboring period. This is what ensures the sensitivity of the motor to the asymmetry of the spatially periodic potential profile. A temperature-related quantity may be not only the intensity of the motor effect itself, i.e. the value of the average particle flux, which determines the average velocity of a ratchet, but also its direction [7]. Thus, though the average velocity of a Brownian motor is a complex quantity that depends on many parameters of the system, in most model and real systems the direction of motor motion depends on both the results of competition between the spatial and temporal asymmetries of the components of the fluctuating nanoparticle potential energy and temperature $[7,10,45]$.

Theoretical study of Brownian ratchets involves analytical solving the diffusion dynamics equations or numerical modeling based on them $[5,46,47]$. In the overwhelming majority of problems, nonequilibrium fluctuations of the characteristics of a nanoparticle or external fields are introduced through specifying a time dependence of the nanoparticle potential energy. Obtaining analytical expressions for the average motor velocity, $\langle v\rangle$, a nonzero value of which determines the presence of the ratchet effect, is a difficult task. It implies finding a solution to the corresponding equation with periodic boundary conditions and subsequent averaging the solution over the potential energy fluctuations. These steps can be exactly performed only for the simplest, highly idealized, space-time dependences of the driving force $F(x, t)=-\partial U(x, t) / \partial t$ corresponding to the nanoparticle potential energy $U(x, t)[1,9]$. The development of approximations becomes therefore a source of significant advances in developing the ratchet theory. The hightemperature (low-energy) approximation, low-temperature approximation (kinetic approach), adiabatic (low-frequency) approximation, and the approximation of slightly fluctuating potential energy $[5,45,48-53]$ are the most known among approximate approaches. The most fruitful one may be the high-temperature approximation, in which the amplitude of spatial changes in the nanoparticle potential energy is considered small relative to the thermal energy $k_{B} T\left(k_{B}\right.$ is the Boltzmann constant, $T$ is the absolute temperature). An obtaining a general expression 
for the velocity of a high-temperature Brownian motor with an arbitrary spatial dependence of the force $F(x, t)$ made it possible to perform a comparative analysis of behavior of stochastic and deterministic Brownian motors in the entire range of fluctuation frequencies [21,48], and to study the manifestation of the nature of fluctuations in motor characteristics $[11,52]$. The inapplicability of the high-temperature results to most biological ratchets, as well as the obvious impossibility of studying temperature-related effects for medium and low temperatures are the disadvantages of this powerful approximation.

High-temperature Brownian motors with spatially harmonic perturbations of an asymmetric spatially periodic potential are characterized by a multiplicative structure of the expression for the average motor velocity relative to the geometric parameters of the system, temperature, and time parameters of the control signal. First this result, obtained for a pulsating ratchet with a sawtooth stationary potential profile, was presented in [11], where it was used as an example demonstrating the possibility of controlling the motion direction by tuning the phase shift between the controlling spatial harmonic perturbation and the position of the maximum of the stationary part of the profile (determining its asymmetry parameter). The discovered insensitivity of the stopping points of such high-temperature ratchets to the values of frequency and temperature, as well as understanding of the importance of these control parameters in ratchets with complex potential reliefs motivated the studying the ratchet motion modes at arbitrary temperatures. To do that, here we use the approximation of slightly fluctuating potential energy (called hereafter as SFPE approximation), reasonable for describing artificial ratchets, in which the amplitude of an external disturbing signal is, as a rule, much less than that of the potential energy of the particle-substrate interaction. The mathematical apparatus of this approximation is the method of Green's functions, developed in the coordinate representation in $[5,53]$. The second section discusses both the difficulties of applying the general integral relation for the ratchet velocity obtained in the coordinate representation and the ways to overcome those difficulties. The proposed ways made it possible, in particular, to prove the possibility and reveal the mechanisms for controlling the ratchet motion direction by temperature and fluctuation frequency tuning. In the third section, we propose a method for solving the ratchet model, based on the Fourier representation of the governing functional dependences and the sought quantities, and specify the obtained relations for the two main processes governing the changes in the particle potential energy with time. The following sections demonstrate the effectiveness of the proposed method by calculating specific systems. We apply it to study the temperature and frequency controlling of ratchet motion in a potential profile of a complex shape that occurs when a spatially periodic sawtooth potential relief is perturbed by a small harmonic signal with a tunable phase shift. We discuss the role of temperature and frequency as parameters controlling the intensity and direction of the ratchet effect.

\section{Approximation of Slightly fluCtuating potential EnERGy}

The SFPE approximation was proposed to describe a variety of real and model ratchet systems and was developed in $[5,53]$. It assumes that the potential energy $U(x, t)$ entering into the Smoluchowski equation for the distribution function $\rho(x, t)$ of the nanoparticle coordinate

$$
\begin{gathered}
\frac{\partial}{\partial t} \rho(x, t)+\frac{\partial}{\partial x} J(x, t)=0, \\
J(x, t)=-D e^{-\beta U(x, t)} \frac{\partial}{\partial x} e^{\beta U(x, t)} \rho(x, t),
\end{gathered}
$$

has an additive-multiplicative form:

$$
U(x, t)=u(x)+\sigma(t) w(x),
$$


in which the first term $u(x)$ describes a stationary (unperturbed) profile, and the second term $\sigma(t) w(x)$ describes a fluctuation component (perturbation). Here it is assumed that $|\sigma(t) w(x)| / k_{B} T<<1$, and the values of the function $\sigma(t)$ are of the order of unity, so that the order of the correction $\sigma(t) w(x)$ is determined by the order of the function $w(x)$. Equation (2) is the definition of the probability flux $J(x, t)$, in which $D \equiv(\beta \zeta)^{-1}$ and $\zeta$ are the diffusion coefficient and the friction coefficient, respectively, and $\beta=\left(k_{B} T\right)^{-1}$. Note that, in the SFPE approximation, the ratio $|u(x)| / k_{B} T$ is assumed to be arbitrary, and the transition to the inequality $|u(x)| / k_{B} T<<1$ means the transition to the high-temperature approximation.

For ratchet systems, the force $F(x, t)=-\partial U(x, t) / \partial x$ averaged over $x$ and $t$ must be equal to zero (unbiased driving), which means that equality to zero is also assumed for the mean value of the function $\sigma(t)$ in (3), which plays the role of a fluctuation variable, $\langle\sigma(t)\rangle=0$; symbol $\langle\ldots\rangle$ means the operation of averaging over fluctuations, the definition of which depends on the nature of the quantity $\sigma(t)[5,11]$. Two main classes of Brownian motors, namely pulsating ratchets and tilting ratchets [1], are also described by the form (3) with periodic $u(x+L)=u(x)$ and $w^{\prime}(x) \equiv d w(x) / d x, w(x+L)=w(x)$ for the first class and $w(x)=F x, F=$ const for the second.

The main result of the SFPE approximation is the following integral representation of the average particle flux $J \equiv\langle J(x, t)\rangle$ in the steady state regime of motion $[5,53]$ :

$$
J=(\beta D)^{2} \int_{0}^{L} d x q(x) w^{\prime}(x) \int_{0}^{L} d x^{\prime} S\left(x, x^{\prime}\right) \frac{\partial}{\partial x^{\prime}} w^{\prime}\left(x^{\prime}\right) \rho^{(0)}\left(x^{\prime}\right) .
$$

This flux sets the average velocity of the Brownian ratchet as $\langle v\rangle=L J$, where $L$ is the spatial period of the function $U(x, t)$. In formula (4), the function $\rho^{(0)}(x)$ describes the equilibrium distribution in the stationary potential $u(x), \rho^{(0)}(x)=e^{-\beta u(x)} / \int_{0}^{L} d x e^{-\beta u(x)}, q(x)$ coincides with $\rho^{(0)}(x)$ up to the replacement $u(x)$ by $-u(x)$, and the function $S\left(x, x^{\prime}\right)$ of two spatial variables is defined in terms of the correlation function $K\left(t-t^{\prime}\right) \equiv\left\langle\sigma(t) \sigma\left(t^{\prime}\right)\right\rangle$ and the retarded Green's function $g\left(x, x^{\prime}, t\right)\left[g\left(x, x^{\prime}, t\right)=0\right.$ at $\left.t<0\right]$ as

$$
S\left(x, x^{\prime}\right) \equiv \int_{0}^{\infty} d t g\left(x, x^{\prime}, t\right) K(t) .
$$

The function $g\left(x, x^{\prime}, t\right)$ describes diffusion in the stationary potential relief $u(x)$ and satisfies the equation

$$
\frac{\partial}{\partial t} g\left(x, x^{\prime}, t\right)+\frac{\partial}{\partial x} \hat{J}(x) g\left(x, x^{\prime}, t\right)=-\delta\left(x-x^{\prime}\right) \delta(t)
$$

with a flux operator $\hat{J}(x)$ of the following form

$$
\hat{J}(x)=-D e^{-\beta u(x)} \frac{\partial}{\partial x} e^{\beta u(x)} .
$$

In the cases when the fluctuations of the nanoparticle potential energy are a stochastic dichotomous process $\sigma(t)$ in which $\sigma(t)= \pm 1$ and $K(t)=\exp (-\Gamma|t|)$, where $\Gamma$ is the inverse correlation time, the function $S\left(x, x^{\prime}\right)$ can be interpreted (based on (5)) as the Laplace transform of the Green's function $g\left(x, x^{\prime}, t\right)$. In this case, the equation for finding $S\left(x, x^{\prime}\right)$ has the form

$$
\left[\frac{d}{d x} \hat{J}(x)+\Gamma\right] S\left(x, x^{\prime}\right)=-\delta\left(x-x^{\prime}\right) .
$$

The last is easily obtained from equation (6), if we multiply both parts of (6) by $\exp (-\Gamma t)$ and then integrate the result over $t$ from $-\varepsilon$ to $\infty$, where $\varepsilon$ is an infinitely small positive value; 
the contribution $\exp (\Gamma \varepsilon) g\left(x, x^{\prime},-\varepsilon\right)$ will be zero since $g\left(x, x^{\prime}, t\right)$ is the retarded Green's function. Here the physical meaning of the function $S\left(x, x^{\prime}\right)$ is interpreted as the probability density of finding a particle at a point $x$ in a potential characterized by a lifetime $\Gamma^{-1}$, provided that at the initial time (i.e., at the time corresponding its transition to a state with a given potential energy profile) the particle was located at a point $x^{\prime}$. Note that in [5], the authors also gave an alternative derivation of the formula for the flux (4) with the function $S\left(x, x^{\prime}\right)$ satisfying equation (8) directly from the Smoluchowski equation with sources and sinks (master equation), which describes just a stochastic dichotomous process and is therefore the basis of stochastic models of Brownian motors $[1,54,55]$. The consideration based on equations (1)-(3) is general and covers both deterministic and stochastic ratchet systems. Thus, the SFPE approximation made it possible to use the powerful apparatus of theoretical physics, namely, the apparatus of Green's functions, to study the mechanisms and characteristic features of ratchet functioning. This approach, based on the analysis of expression (4), gave a wide range of analytical results [5].

Though the methods for solving equations of the form (6) or (8) are well known, obtaining analytical results for specific profiles of $u(x)$ (not the simplest model profiles, but complex ones, actual both for practical applications and for generalized theoretical conclusions) turns out to be extremely cumbersome. An example here is the explicit form of the function for a "simple" sawtooth relief $u(x)$ [5]. But even if the function $S\left(x, x^{\prime}\right)$ can be specified analytically, the subsequent taking of the integrals in (4) is also not a trivial problem. Moreover, when using numerical methods for calculating proper integrals, difficulties will arise both in the lowfrequency limit $(\Gamma \rightarrow 0)$ and at the cusp points of the disturbance profiles $w(x)$. Therefore, using the Fourier representation turned out to be a promising alternative to integral relations.

\section{Using the Fourier REPRESENTATION}

In this section, we will propose a method for calculating the characteristics of a Brownian motor with a slightly fluctuating potential profile, based on the Fourier representation of the governing functions and the desired quantities. Rewrite expression (4) for the particle flux in an equivalent form by integrating by parts in the inner integral:

$$
J=-(\beta D)^{2} \int_{0}^{L} d x q(x) w^{\prime}(x) \int_{0}^{L} d x^{\prime} w^{\prime}\left(x^{\prime}\right) \rho^{(0)}\left(x^{\prime}\right) \frac{\partial}{\partial x^{\prime}} S\left(x, x^{\prime}\right) .
$$

As the functions $q(x), w(x), \rho^{(0)}(x)$ are periodic, we can write them in the form of Fourier series $f(x)=\sum_{p} f_{p} e^{i k_{p} x}$ (where $k_{p}=2 \pi p / L, p=0, \pm 1, \pm 2, \ldots ; f(x)$ means any of these functions). A similar representation for the function of two spatial variables $S\left(x, x^{\prime}\right)$ is

$$
S\left(x, x^{\prime}\right)=\sum_{p p^{\prime}} S_{p p^{\prime}} e^{i k_{p} x-i k_{p^{\prime}} x}
$$

so expression (9) in Fourier components becomes:

$$
J=-i(\beta D L)^{2} \sum_{p p^{\prime} p_{1} p_{2}} k_{p^{\prime}} k_{-p-p_{1}} k_{-p_{2}+p^{\prime}} q_{p_{1}} \rho_{p_{2}}^{(0)} S_{p p^{\prime}} w_{-p-p_{1}} w_{-p_{2}+p^{\prime}}
$$

Thus, we can calculate the average velocity of a Brownian ratchet driven by small fluctuations (stochastic or deterministic periodic) of a spatially periodic potential profile by summation according to (11), provided that the matrix elements $S_{p p^{\prime}}$ are known. Obviously, the generality of formula (11) is the same as that of integral expression (4), i.e. dependences $u(x)$ and $w(x)$ can be any periodic with a period $L$, and $\sigma(t)$ is either a periodic function with a period $\tau$ (in a deterministic process) or given by a correlation function with a correlation time $\Gamma^{-1}$ associated with the average period of the process through the relation $\tau=4 / \Gamma$ (if it describes a stochastic process). In analysis of particular models and calculating their characteristics, difficulties in 
using (11) can arise when one intends to investigate the functioning of ratchets with potential reliefs having areas with large gradients. Jump-like changes in the relief require a quadruple summation over a large number of harmonics. In all other cases, calculations according to (11), as a rule, are more efficient than those according to (4), (9).

The main quantities in (11) are the matrix elements $S_{p p^{\prime}}$. Now, construct a matrix analogue of equation (6) and obtain formulas for calculating $S_{p p^{\prime}}$. From the definition (5), the matrix elements $g_{p p^{\prime}}(t)$ in the Fourier representation of the retarded Green's function

$$
g\left(x, x^{\prime}, t\right)=\sum_{p p^{\prime}} g_{p p^{\prime}}(t) e^{i k_{p} x-i k_{p^{\prime}} x^{\prime}}
$$

relate to $S_{p p^{\prime}}$ as

$$
S_{p p^{\prime}}=\int_{0}^{\infty} d t g_{p p^{\prime}}(t) K(t)
$$

Therefore, when $g_{p p^{\prime}}(t)$ are known, to go to $S_{p p^{\prime}}$ one should only specify the correlation functions, i.e. specify the processes that control the change in potential energy with time.

Substituting (12) into (6) gives the following equation for finding the matrix elements $g_{p p^{\prime}}(t)$

$$
\left(\frac{\partial}{\partial t}+D k_{p}^{2}\right) g_{p p^{\prime}}(t)+\beta D k_{p} \sum_{\tilde{p}} k_{p-\tilde{p}} u_{p-\tilde{p}} g_{\tilde{p} p^{\prime}}(t)=-\frac{1}{L} \delta_{p p^{\prime}} \delta(t) .
$$

We can represent (14) in a quite compact form (convenient to write the general solution) if define a new operator $\hat{A}$ with its matrix elements $A_{p \tilde{p}} \equiv D k_{p}^{2} \delta_{p \tilde{p}}+\beta D k_{p} k_{p-\tilde{p}} u_{p-\tilde{p}}$ :

$$
\frac{\partial}{\partial t} \hat{g}(t)+\hat{A} \hat{g}(t)=-\frac{1}{L} \hat{1} \delta(t)
$$

where $\hat{1}$ is the unit operator with matrix elements $\delta_{p p^{\prime}}$. It's solution is the operator

$$
\hat{g}(t)=-\frac{1}{L} \theta(t) e^{-\hat{A} t}
$$

We can use it to construct the desired operator $\hat{S}$, provided that the process described by $\sigma(t)$ is given, i.e. we know the correlation function $K(t)$ :

$$
\hat{S}=\int_{0}^{\infty} d t \hat{g}(t) K(t)=-\frac{1}{L} \int_{0}^{\infty} d t K(t) e^{-\hat{A} t}
$$

Next, we consider two examples of such a construction by specifying processes that govern the change in potential energy. Choose the processes that are most important in the description and design of Brownian ratchets. We start with a dichotomous Markov stochastic process, for which $\sigma(t)$ takes two values +1 and -1 , with given transition rates $\gamma_{+}$and $\gamma_{-}$. Typical systems, modeled as ratchets with such a time dependence of driving forces, are protein (molecular) motors, alternately living in two conformational states with different coordinate profiles of the potential energy of the globular head-track interaction [22,44]. For a Markov dichotomous process, the correlation function $K(t)$ is exponential, $K(t)=e^{-\Gamma t}$, and the sum of the transition rates determines the inverse correlation time $\Gamma=\gamma_{+}+\gamma_{-}$. Then, according to (17), the operator $\hat{S}$ becomes 


$$
\hat{S}=-\frac{1}{L}(\hat{A}+\Gamma \hat{1})^{-1}
$$

that is, to find $S_{p p^{\prime}}$, one should solve the equation

$$
\sum_{\tilde{p}}\left[\left(D k_{p}^{2}+\Gamma\right) \delta_{p \tilde{p}}+\beta D k_{p} k_{p-\tilde{p}} u_{p-\tilde{p}}\right] S_{\tilde{p} p^{\prime}}=-\frac{1}{L} \delta_{p p^{\prime}}
$$

Thus, we get a principal result which optimizes the discussed mathematical model: with given coordinate dependence of the stationary potential component $u(x)$, the problem of solving differential equation (8) is reduced to the problem of the matrix inverse $[\hat{A}+\Gamma \hat{1}]$. Modern computational mathematics suggests a large set of effective methods to solve this problem, including methods applicable at large number of harmonics $u_{p-\tilde{p}}$, i.e. at large size of the matrix of the operator $[\hat{A}+\Gamma \hat{1}]$.

As a second example we choose the periodic processes of changes in the nanoparticle potential energy with time. As a rule, they are typical for artificial ratchet systems. Then $\sigma(t+\tau)=\sigma(t)=$ $\sum_{j} \sigma_{j} \exp \left(-i \omega_{j} t\right)$, where $\tau$ is the period $\omega_{j}=2 \pi j / \tau, j=0, \pm 1, \pm 2, \ldots$, and $\sigma_{j}$ is the Fourier component of $\sigma(t)$. The averaging operation for such processes, $\langle\ldots\rangle$, implies averaging over the period $\left(\tau^{-1} \int_{0}^{\tau} d t \ldots\right)$, so $\langle\sigma(t)\rangle=\sigma_{0}=0$, and the correlation function $K(t)=\sum_{j}\left|\sigma_{j}\right|^{2} e^{-i \omega_{j} t}$. Then, according to (17), the operator $\hat{S}$ is

$$
\hat{S}=-\frac{1}{L} \sum_{j}\left|\sigma_{j}\right|^{2} \int_{0}^{\infty} d t e^{-\left(\hat{A}+i \omega_{j} \hat{1}\right) t}=-\frac{1}{L} \sum_{j}\left|\sigma_{j}\right|^{2}\left[\hat{A}+i \omega_{j} \hat{1}\right]^{-1} .
$$

Obviously, in the description of deterministic ratchets, the matrix $\left[\hat{A}+i \omega_{j} \hat{1}\right]$ is similar to $[\hat{A}+\Gamma \hat{1}]$ in the stochastic description (cf. (20) and (18)) up to the replacement of the inverse correlation time $\Gamma$ by $i \omega_{j}$. Since $\left|\sigma_{-j}\right|^{2}=\left|\sigma_{j}\right|^{2}$, one can rewrite the operator (20) in an equivalent real form:

$$
\hat{S}=-\frac{1}{L} \hat{A} \sum_{j}\left|\sigma_{j}\right|^{2}\left[\hat{A}^{2}+\omega_{j}^{2}\right]^{-1}
$$

The matrix elements of the operator $\hat{A}^{2}$ can be calculated as

$$
\left(\hat{A}^{2}\right)_{p p^{\prime}}=D^{2} k_{p}^{4} \delta_{p p^{\prime}}+\beta D^{2} k_{p} k_{p-p^{\prime}}\left(k_{p}^{2}+k_{p^{\prime}}^{2}\right) u_{p-p^{\prime}}+\beta^{2} D^{2} k_{p} \sum_{\tilde{p}} k_{\tilde{p}} k_{p-\tilde{p}} k_{\tilde{p}-p^{\prime}} u_{p-\tilde{p}} u_{\tilde{p}-p^{\prime}}
$$

Thus, within the Fourier representation, the main relations of the SFPE approximation are given by the formula (11) for the flux, with the matrix elements $S_{p p^{\prime}}$ of the operator $\hat{S}$ calculated as inverse matrices through (18) and (21) for stochastic dichotomous and deterministic periodic processes, respectively. 
d. V. SHAPOCHKINA ${ }^{A}$, T. YE. KOROCHKOVA ${ }^{B}$, V. M. ROZENBAUM $^{B}$, A. S. BUGAEV ${ }^{C}$, L. I. TRAKHTENBERG ${ }^{C, D}$

\section{Temperature-Frequency CONTROLling THE RATCHET OPERATing MOdeS}

One of the effective methods of controlling the intensity and direction of the ratchet effect is to use a spatially harmonic signal as an external forcing the ratchet to move [11]. For the additive-multiplicative form (3) of the particle potential energy, such a forcing can be represented as

$$
w(x)=w \cos \left[2 \pi\left(x / L-\lambda_{0}\right)\right] .
$$

The harmonic signal $w(x)$ of amplitude $w$ and phase shift $\lambda_{0}$ is a reasonable model of external perturbations in many cases when the perturbations are of an artificial nature. As a stationary spatially periodic potential, a convenient choice is a piecewise linear sawtooth profile

$$
u(x)=u\left\{\begin{array}{l}
x / l, 0<x<l, \\
(L-x) /(L-l), \quad l<x<L,
\end{array}\right.
$$

characterized by the barrier $u$ and the widths of the links $l$ and $L-l$. It is a good model of the interaction of a nanoparticle with a track (polar substrate, microtubule, filament, etc.) and is a reasonable approximation for many real motor systems; it is also the most often choice of the geometry of experimental patterns $[17,18,30,38,41,56]$. At the same time, it turns out to be a fruitful approximation to reveal and describe a number of subtle mechanisms responsible for ratchet functioning: the level of "sensitivity" of motors of different classes to the presence of sharp inflection points in the spatial dependence of the potential energy, the influence of the potential profile asymmetry and competition of characteristic times on the ratchets' characteristics and their operation modes, etc. [10,21,52,57]. For fluctuations described by a stochastic dichotomous process, the potential energy fluctuates between two profiles $u(x) \pm w(x)$ (fig. 1).

From [11], for a high-temperature ratchet with a potential profile defined by (3), (23), (24) $\left(|U(x, t)|<<k_{B} T\right)$ and undergoing stochastic dichotomous fluctuations with inverse correlation time $\Gamma$, the fluctuation frequency, temperature, and the model geometry (the asymmetry of the stationary component $u(x)$, specified by $\lambda \equiv l / L$ and the phase shift $\lambda_{0}$ of the disturbing signal $w(x)$ ) affect the ratchet average velocity independently. That independence is in the following multiplicative representation:

$$
\begin{aligned}
& J / J_{0}=-\pi \alpha^{2} f_{1}(\alpha \gamma) f_{2}\left(\lambda, \lambda_{0}\right), \quad J_{0}=(w / u)^{2} \tau_{s l}^{-1}, \quad \tau_{s l}=\zeta L^{2} / u, \\
& \alpha=\beta u, \quad \gamma=\Gamma \tau_{s l}, \quad f_{1}(z)=\frac{z}{\left(4 \pi^{2}+z\right)^{2}}, \quad f_{2}\left(\lambda, \lambda_{0}\right)=\frac{\sin 2 \pi \lambda \cos 2 \pi\left(\lambda-2 \lambda_{0}\right)}{\lambda(1-\lambda)} .
\end{aligned}
$$

Here, the parameters $\alpha$ and $\gamma$ are, respectively, the dimensional reciprocal temperature and the fluctuation frequency. Energy is measured in units of the barrier $u$, and time is measured in units of the characteristic sliding time $\tau_{s l}$ along the potential $u(x)$. While the positively defined factor $\alpha^{2} f_{1}(\alpha \gamma)$ sets the temperature-frequency dependence, the function $f_{2}\left(\lambda, \lambda_{0}\right)$ changes sign at $\lambda-2 \lambda_{0}= \pm 1 / 4, \pm 3 / 4$. This sets the stopping points of such a ratchet, which are independent both of temperature and frequency.

It is reasonable to assume that outside the high-temperature approximation, the stopping points will depend both on temperature and frequency, that will permit the temperaturefrequency controlling the ratchet. Next, we use the just developed calculating scheme based on the Fourier representation within the SFPE approximation to test this assumption. The amplitude of the spatially harmonic perturbation $w$ is fixed small as compared with the thermal energy $k_{B} T$, while we allow the barrier of the sawtooth potential $u$ to be arbitrary. To perform calculations by (11), (19), we use the Fourier components of the functions $w(x)(23)$ and $u(x)$ (24) which are given by the following expressions:

$$
w_{p}=\frac{w}{2}\left[e^{2 \pi i \lambda_{0}} \delta_{p,-1}+e^{-2 \pi i \lambda_{0}} \delta_{p, 1}\right], \quad u_{p}=u \frac{e^{-2 \pi i \lambda p}-1}{4 \pi^{2} p^{2} \lambda(1-\lambda)} .
$$


The presence of the Kronecker $\delta$ in the Fourier component $w_{p}$ simplifies the formula (11) for the desired particle flux $J$ to double summation:

$$
\begin{aligned}
& J=-i(\pi \beta D w)^{2} \sum_{p p^{\prime}} k_{p^{\prime}} S_{p p^{\prime}}\left[q_{-p+1} Z_{p^{\prime}}^{(+)}+q_{-p-1} Z_{p^{\prime}}^{(-)}\right] \\
& Z_{p^{\prime}}^{( \pm)}=e^{ \pm 4 \pi i \lambda_{0}} \rho_{p^{\prime} \pm 1}^{(0)}-\rho_{p^{\prime} \mp 1}^{(0)} .
\end{aligned}
$$

Here, the matrix elements $S_{p p^{\prime}}$ are found by solving equation (19), the component $q_{p}$ has the form

$$
q_{p}=\frac{1}{L} \frac{e^{\alpha-2 \pi i \lambda p}-1}{e^{\alpha}-1} \frac{\alpha^{2}}{\alpha^{2}+2 \pi i(1-2 \lambda) \alpha p+4 \pi^{2} \lambda(1-\lambda) p^{2}}
$$

and $\rho_{p}^{(0)}$ follows from (28) by the replacement $\alpha \rightarrow-\alpha$.

Figure 2 gives the temperature-frequency dependences of the flux, which determines the average velocity of the considered Brownian ratchet, at various values of the phase shift $\lambda_{0}$, calculated from the above relations. The frequency dependences (fig. 2 a) show that, for example, at sufficiently large $\alpha$ values, $\alpha=5$ (that is, outside the region of the high-temperature approximation with $\alpha<<1$ ), the stopping points appear at certain frequencies which depend on the phase shift $\lambda_{0}$. Temperature dependences (fig. $2 \mathrm{~b}$ ), calculated for the same $\lambda_{0}$ values and at two frequencies $\gamma=3.98$ and 63.1 (open and filled markers, respectively), show that, indeed, the stopping points occur only at sufficiently low temperatures (large $\alpha$ values) and at not very high frequencies. This conclusion is in good agreement with the results of the well-known high-frequency approximation (see formula (27) in [5]), according to which at $\gamma>>1$ the flux is

$$
J / J_{0}=-\frac{\pi \alpha^{3}}{4 \gamma \sinh ^{2}(\alpha / 2)} f_{2}\left(\lambda, \lambda_{0}\right),
$$

and its sign is determined by the sign of the function $f_{2}\left(\lambda, \lambda_{0}\right)$, which is the same as in the high-temperature formula (25). In this case, when $\alpha<<1$, the factor at $f_{2}\left(\lambda, \lambda_{0}\right)$ is equal to $-\pi \alpha / \gamma$, that is, it coincides with the factor arising in (25) when $\gamma>>1$, as it should be. Thus, the high-frequency wing of the curves in fig. 2 (a) is in good agreement with the high-frequency asymptotics (29) at $\alpha=5$; similarly, in fig. 2 (b), the left wings (at $\alpha<1$ ) are well described by the high-temperature formula (25).

The fact that with decreasing temperature (increasing $\alpha$ ) the stopping points become dependent on both temperature and frequency is illustrated by the diagram in fig. 3 plotted for $\lambda=0.1$ and $\lambda_{0} \in(0,0.5)$. In the region of small $\alpha$ values, stopping points arise at $\lambda_{0}=0.175$ and 0.425 , and the particles will move to the right when the phase shift values are within the interval $(0.175,0.425)$. With an increase $\alpha$, the region of positive flux values is displaced, and this displacement is the greater the smaller the $\gamma$ values. When $\gamma \rightarrow \infty$ there is no displacement at all, and the entire region $(0.175,0.425)$ belongs to positive fluxes, as in case of $\alpha<<1$.

Thus, the results of numerical calculations confirm that, outside the high-temperature approximation, one obtains new possibilities to govern the flux direction: by changing both the frequency and temperature.

\section{Discussion AND CONCLUSiOnS}

In previous works, the authors proposed a new approximation of slightly fluctuating potential energy $[5,53]$, in which the potential energy is represented as the sum of a stationary relief and a small fluctuation component. In contrast to the high-temperature approximation, in which both of these contributions were small relative to $k_{B} T$, this SFPE approximation is promising for describing the functioning of artificial nanomotors, since it allows, in particular, to study the 
characteristics of the ratchet effect in a real temperature range. Among them are, for example, temperature dependences of the motion parameters, not limited by the trivial proportionality to the inverse cube of temperature following from the high-temperature approach. Within the developed approximation, the main step is to find the Green's function of diffusion in the stationary component of the potential, which is a response of the system to a delta-shaped spacetime perturbation. Then, with its help, the ratchet effect is described directly as a result of the action of a small perturbation. Obtaining an analytical expression for the Green's function is a tough problem realizable only for a narrow set of potential reliefs of a piecewise linear shape. The subsequent calculating the ratchet characteristics involves numerical taking of double integrals. For wide using the approximation of small fluctuations, one should therefore develop numerical methods that would make it possible to calculate the Green's function, not just the characteristics of the ratchet effect itself. In this article, we solved the problem by the Fourier method: finding the Green's function is reduced to the matrix inverse in the space of Fourier harmonics, and the subsequent integrations are replaced by summations of different multiplicity depending on the spatial geometry of the system.

To illustrate the application of the proposed method, we analyzed a ratchet operating in a sawtooth potential with an arbitrary value of the barrier-to-thermal-energy ratio under the action of a spatially harmonic perturbation. The theoretical high-temperature analysis of the occurrence of the directed particle flux in such a ratchet system, performed in [11], showed that the system is promising in sense of the possibility to reverse the flux direction by the choice of the phase shift of the harmonic signal. In doing so, the stopping points, in which the motion direction was reversed, were invariant with respect to both temperature and the fluctuation frequency. We can say that there existed a degeneration of the stopping points with respect to these parameters. It was assumed that the breaking the high-temperature approximation can remove the degeneracy, i.e. can introduce the dependence of the stopping points on both temperature and the fluctuation frequency.

The results of this article, obtained within the approximation of small fluctuations, convincingly proved this assumption and showed that the intensity and direction of the ratchet effect in the discussed system can be controlled not only by the phase shift of the harmonic signal, but also by a larger number of parameters. At high fluctuation frequencies, the stopping points are almost independent of temperature and are determined solely by the phase shift, similarly as they do at the high-temperature approximation. With decreasing frequency, in the phase shift-temperature diagram, the region of fluxes of one sign displaces the region of the other sign (fig. 3). Finally, at low frequencies, the displaced region has the smallest area and is characterized by the lowest temperature, above which, for any phase shifts, the fluxes of only one sign can exist. The revealed regularities are a consequence of nonlinear phenomena occurring in complex systems called Brownian ratchets, and give wide possibilities for temperature-frequency control of nanoparticle transport.

\section{Acknowledgments}

This work was carried out within the framework of State Assignment 0082-2018-0003 (registration number AAAA-A18-118012390045-2) and supported by the Russian Foundation for Basic Research (projects 20-57-00007 and 18-29-02012-mk), the Belarusian Republican Foundation for Basic Research (project F20R-032).

\section{REFERENCES}

[1] P. Reimann, Phys. Rep. 361, 57 (2002).

[2] P. Hänggi and F. Marchesoni, Rev. Mod. Phys. 81, 387 (2009).

[3] D. Cubero, F. Renzoni, Brownian Ratchets: From Statistical Physics to Bio and Nano-motors. Cambridge: Cambridge University Press (2016). 
[4] B. Lau, J. Kedem, D. Schwabacher, D. Kwasnieski, and E. A. Weiss, Mater. Horiz. 4, 310, (2017).

[5] V. M. Rozenbaum, I.V. Shapochkina, L.I. Trakhtenberg Physics-Uspekhi. 62(5), 496 (2019).

[6] Yu. V. Gulyaev, A. S. Bugaev, V. M. Rozenbaum and L. I. Trakhtenberg, Phys. Usp. 63 (4), 31, (2020).

[7] N.Yu. Shved, I.V.Shapochkina, V.M. Rosenbaum, Vestnik BGU. 2014. 1(2): 27.

[8] V. M. Rozenbaum, Yu. A. Makhnovskii, I. V. Shapochkina, S.-Y. Sheu, D.-Y. Yang, and S. H. Lin, Phys. Rev. E 92, 062132 (2015).

[9] V. M. Rozenbaum, T. Y. Korochkova, D.-Y. Yang, S. H. Lin, and T. Y. Tsong, Phys. Rev. E 71, 041102 (2005).

[10] V. M. Rozenbaum, T. Y. Korochkova, A. A. Chernova, and M. L. Dekhtyar, Phys. Rev. E 83, 051120 (2011).

[11] V. M. Rozenbaum, I. V. Shapochkina, Y. Teranishi, L. I. Trakhtenberg, Phys. Rev. E 99(1), 012103 (2019)

[12] P. Reimann, Phys. Rev. Lett. 86, 4992 (2001).

[13] S. Denisov, S. Flach, P. Hänggi, Phys. Rep. 538, 77 (2014).

[14] D. Cubero, F. Renzoni, Phys. Rev. Lett. 116, 010602 (2016).

[15] V. M. Rozenbaum, I. V. Shapochkina, Y. Teranishi, and L. I. Trakhtenberg, Pis'ma Zh. Eksp. Teor. Fiz. 107, 525 (2018).

[16] V. M. Rozenbaum, I. V. Shapochkina, Y. Teranishi, and L. I. Trakhtenberg. Phys. Rev. E 100, 022115 (2019).

[17] J. S. Roth. Y. Zhang, P. Bao, M. R. Cheetham, X. Han and S. D. Evans. Appl. Phys. Lett. 106, 183703 (2015).

[18] M. R. Cheetham, J. P. Bramble, D. G. G. McMillan, R. J. Bushby, P. D. Olmsted, L. J. C. Jeuken, and S. D. Evans. Soft Matter. 8, 5459 (2012).

[19] C. F. Chou, O. Bakajin, S. W. P.Turner, T. A. J. Duke, S. S. Chan, E. C. Cox, H. G. Craighead, and R. H. Austin, Proc. Natl. Acad. Sci. U.S.A. 96, 13762 (1999).

[20] S. Matthias, and F. Müller, Nature 424, 53 (2003).

[21] V. M. Rozenbaum, I. V. Shapochkina, S.-Y. Sheu, D.-Y. Yang, S. H. Lin, Phys. Rev. E 94(5), 052140 (2016).

[22] P. M. Hoffmann, Rep. Prog. Phys. 79(3), 032601 (2016).

[23] S. Erbas-Cakmak, D. A. Leigh, C. T. McTernan, and A. L. Nussbaumer, Chem. Rev. 115, 10081 (2015).

[24] G. Tsiavaliaris, S. Fujita-Becker, and D.J. Manstein, Nature 427, 558561 (2004).

[25] M. Nakamura, L. Chen, S. C. Howes, T. D. Schindler, E. Nogales, and Z Bryant, Nature Nanotech. 9, 693 (2014).

[26] N. Koumura, R. W. J. Zijlstra, R. A. van Delden, N. Harada, and B. L. Feringa, Nature 401, 152 (1999).

[27] J. Berna, D. A. Leigh, M. Lubomska S. M. Mendoza, E. M. Perez, P. Rudolf, G. Teobaldi and F. Zerbetto, Nature Mater. 4, 704 (2005).

[28] V. Serreli, C. F. Lee, E. R. Kay, and D. A. Leigh, Nature 445, 523 (2007).

[29] . H. Linke, T. E. Humphrey, A. L“ofgren, A. O. Sushkov, R. Newbury, and R. P. Taylor, Science 286, 2314 (1999).

[30] C.-S. Lee, B. Janko, I. Derenyi, and A.-L. Barabasi, Nature 400, 337 (1999).

[31] D. Cole, S. Bending, S. Savel'ev, A. Grigorenko, T. Tamegai, F. Nori, Nature mat. 5, 305 (2006).

[32] C. C. de Souza Silva, J. V. de Vondel, M. Morelle, and V. V. Moshchalkov, Nature 440, 651 (2006).

[33] K. Yu, T. W. Heitmann, C. Song, M. P. DeFeo, B. L. T. Plourde, M. B. S. Hesselberth, and P. H. Kes, Phys. Rev. B 76, 220507 (2007).

[34] D. E. Shalom, and H. Pastoriza, Phys. Rev. Lett. 94, 177001 (2005).

[35] J. Rousselet, L. Salome, A. Ajdari, and J. Prost, Nature 370, 446 (1994).

[36] J. S. Bader, R. W. Hammond, S. A. Henck, M. W. Deem, G. A. McDermott, J. M. Bustillo, J. W. Simpson, G. T. Mulhern, J. M. Rothberg, Proc. Natl. Acad. Sci. U.S.A. 96, 13165 (1999).

[37] S.-H. Lee, K. Ladavac, M. Polin, and D. G. Grier, Phys. Rev. Lett. 94, 110601 (2005).

[38] W. C. Germs, E. M. Roeling, L. J. van Ijzendoorn, B. Smalbrugge, T. de Vries, E. J. Geluk, R. A. J. Janssen, and M. Kemerink, Phys. Rev. E: Stat., Nonlinear, Soft Matter Phys. 86, 041106 (2012).

[39] S.-H. Wu, N. Huang, E. Jaquay, and M. L. Povinelli, Nano Lett. 16, 5261 (2016)

[40] S. Matthias and F. Muller, Nature 424, 53 (2003).

[41] R. D. Astumian, Science 276, 917 (1997).

[42] V. M. Rozenbaum, D.-Y. Yang, S. H. Lin, T. Y. Tsong, J. Phys. Chem. B. 108(40), 15880 (2004).

[43] S. P. Gilbert, M. R. Webb, M. Brune, K. A. Johnson, Nature 373, 671 (1995).

[44] C. L. Asbury, A. N. Fehr, and S. M. Block, Science 302, 2130 (2003).

[45] V. M. Rozenbaum, JETP Letters 88, 342-346 (2008).

[46] H. Risken The Fokker-Plank Equation. Methods of Solution and Applications. Springer, Berlin, 1984. p. 472.

[47] I. Goychuk, Adv. Chem. Phys. 150, 187 (2012).

[48] V. M. Rozenbaum, JETP, 110 (4), 653 (2010).

[49] J.M.R. Parrondo, Phys. Rev. E 57, 7297 (1998).

[50] V. M. Rozenbaum, I. V. Shapochkina JETP Letters 102, 248-253 (2015). 
[51] V. M. Rozenbaum, Yu. A. Makhnovskii, I. V. Shapochkina, S.-Y. Sheu, D.-Y. Yang, and S. H. Lin, Phys. Rev. E. 85, 041116 (2012).

[52] I. V. Shapochkina, V. M. Rozenbaum, S.-Y. Sheu, D.-Y. Yang, S. H. Lin, and L. I. Trakhtenberg, Physica A $514,71(2019)$.

[53] V. M. Rozenbaum, I. V. Shapochkina, S. H. Lin, and L. I. Trakhtenberg, JETP Letters 105, 8, 542 (2017).

[54] K. V. Gardiner, Stochastic Methods in Natural Science [Russian translation], Mir, Moscow (1986).

[55] C. Van den Broeck and P. Hänggi, Phys. Rev. A 30:5 2730 (1984).

[56] V. M. Rozenbaum, Yu. A. Makhnovskii, I. V. Shapochkina, S.-Y. Sheu, D.-Y. Yang, and S. H. Lin, Phys. Rev. E 89, 052131 (2014).

[57] V. M. Rozenbaum, Yu. A. Makhnovskii, I. V. Shapochkina, S.-Y. Sheu, D.-Y. Yang, and S. H. Lin, Phys. Rev. E, 92 (5), 062132 (2015).

\section{Figure CAPtions}

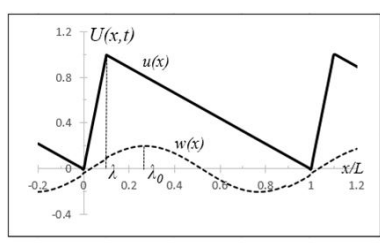

FiguRE 1. Additive-multiplicative contributions to the ratchet potential energy $U(x, t)$, which includes a stationary sawtooth relief $u(x)$ and a fluctuating small harmonic component $w(x)$, characterized, respectively, by the asymmetry parameter $\lambda=l / L$ and phase shift $\lambda_{0}$ (relations (3), (23), (24)).
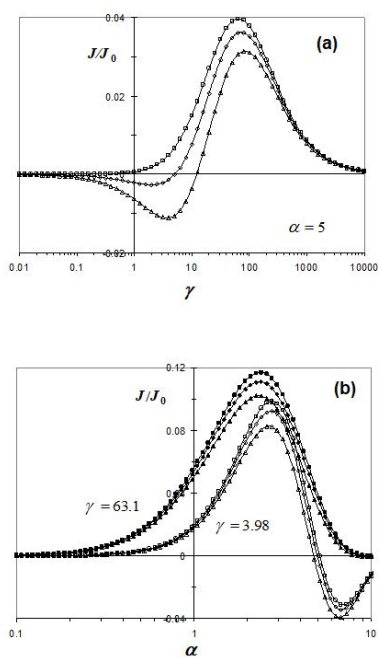

Figure 2. The flux $J$, measured in units $J_{0}=\left(w_{0} / u_{0}\right)^{2} \tau_{s l}^{-1}$, vs the dimensionless fluctuation frequency $\gamma=\Gamma \tau_{s l}$ at $\beta u_{0}=5$ (a) and vs dimensionless inverse temperature $\alpha=\beta u_{0}$ (b) for different values of the phase shift of the spatially harmonic signal $\lambda_{0}=0.215,0.218,0.220$ (curves with triangular, rhombic, and square markers, respectively). The value of the asymmetry parameter of the sawtooth potential is $\lambda=0.1$. 
TEMPERATURE-FREQUENCY CONTROLLING THE CHARACTERISTICS OF A PULSATING BROWNIAN RATCHET WITH SLIC

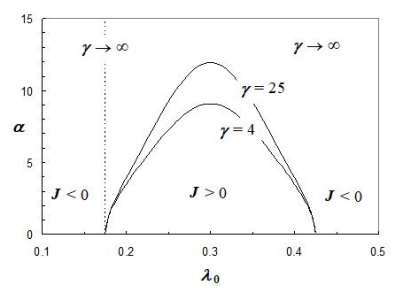

Figure 3. Phase diagram of the parameters $\alpha$ and $\lambda_{0}$, specifying at $\lambda=0.1$ the range of values that correspond to particle motion to the right (inner area with the base $\left.0.175<\lambda_{0}<0.425\right)$ and to the left. Frequency values $\gamma$ are indicated on the corresponding curves. 